\title{
Towards the Development of a Brain Semi-controlled Wheelchair for Navigation in Indoor Environments
}

\author{
Hailah AlMazrua ${ }^{1}$, Abir Benabid Najjar ${ }^{2}$ \\ College of Computer and Information Science \\ King Saud University, Riyadh \\ Saudi Arabia
}

\begin{abstract}
Several technological advancements emerged providing the technical assistance supporting people with special needs in tackling their everyday tasks. Particularly, with the advancements in cost-effective Brain-Computer Interfaces (BCI), they can be very useful for people with disabilities to improve their quality of life. This paper investigates the usability of lowcost BCI for navigation in an indoor environment, which is considered one of the daily challenges facing individuals with mobility impairment. A software framework is proposed to control a wheelchair using three modes of operations: braincontrolled, autonomous and semi-autonomous, taking into consideration the usability and safety requirements. A prototype system based on the proposed framework was developed. The system can detect an obstacle in the front, right and left sides of the wheelchair and can stop the movement automatically to avoid collation. The usability evaluation of the proposed system, in terms of effectiveness, efficiency and satisfaction, shows that it can be very helpful in the daily life of the mobility impaired people. An experiment was conducted to assess the usability of the proposed framework using the prototype system. Subjects steered the wheelchair using the three different operation modes effectively by controlling the direction of motion.
\end{abstract}

Keywords-Usability; wheelchair navigation; indoor navigation; mobility impairment; obstacle avoidance; obstacle detection; path planning; $\mathrm{BCI}$; brain-computer interaction

\section{INTRODUCTION}

Independent mobility is an important aspect in the quality of life for individuals with mobility impairments. Though the needs of many individuals with mobility impairments can be satisfied with traditional manual or powered wheelchairs, a part of the impaired community finds it difficult and sometimes impossible to use the wheelchairs independently. This part comprises of individuals with low vision, visual field reduction, spasticity, tremors, or cognitive deficits [1]. These individuals have to depend on another person to push them while they are on the wheelchair, as they often lack the independent mobility to control a powered wheelchair due to the nature of their disability. In the U.S, almost $10 \%$ of the legally blind individuals also have a mobility impairment [3], which makes many of them hesitate to visit unfamiliar places since they have no information about the new environment and its accessibility conditions.
Navigation tasks are regarded as one of the critical challenges facing individuals with mobility impairments. According to the Environmental Protection Agency (EPA), the average American spends 93\% of their life indoors [2]. The need to consider indoor navigation is even higher in the Saudi local context, mainly for individuals with some cognitive, visual, or physical impairments since $3.73 \%$ of the population have some form of functional disability [31].

Moreover, the safety, easiness and usability features in the assistive systems are considered a crucial requirement considering the special situation of mobility-impaired individuals. However, the currently available navigational assistive systems lack these features [32]. Highly impaired individuals, using the powered wheelchair, require an autonomous wheelchair for navigation [4]. Therefore, developing a smart wheelchair that would transport the mobility-impaired individuals to their desired destination without their direct control would significantly improve their quality of life, taking into account that such wheelchair has to plan a quick and a safe path even when faced with an obstacle. Thus, providing the mobility-impaired individuals with some level of independence, by not relying on another person for assistance, as the wheelchair would maneuver through the obstacles by itself. Also, providing the wheelchair's rider the ability to control the wheelchair according to his/her preference is a must. Some riders would like to have a full control on how the wheelchair moves, while others prefer to just sit back and make the electric wheelchair moving autonomously.

Furthermore, the obstacle detection is a key component for any autonomous system, in order to ensure the safety of the individual driving it. Therefore, many research studies, according to [36], have considered integrating different types of sensors in their autonomous systems, in order to detect and avoid the faced obstacles. These sensors can either be ultrasonic, infrared, computer vision type sensors, laser sensors, or a combination of different types.

This paper focuses on the usability engineering of an obstacle avoidance system by combining the new technologies with path optimization techniques taking into consideration the special needs of the target users throughout the development cycle. 


\section{BACKGROUND}

Assistive technologies are developed according to two approaches: (1) developing special hardware devices designed for disabled people or (2) using existing hardware devices and integrating specific applications to improve some aspects of the individuals" daily life. The latter approach is what will be used in this paper.

Smart wheelchairs can either be autonomous, nonautonomous or a mix of both types (semi-autonomous). In autonomous wheelchairs, the TetraNauta [5], and Kanazawa University [6] wheelchairs include a computer for processing along with different types of sensors. They tackled specific problems such as: obstacle avoidance, local environment mapping, and path navigation. With the autonomous control feature, the system analyses the environment, plans a navigation path, detects an obstacle, makes decisions, and controls the wheelchair's movements [7]. Moreover, several prototypes of smart wheelchairs have been developed and many research papers have been published in this area like in [8] and [9]. However, most of them have hardware and software architectures developed specifically for a particular wheelchair model and usually they tend to be difficult to configure in order to be used by the physically impaired individuals [1][8].

The capability and nature of the user's disability might become a restriction when operating a wheelchair. Therefore, the spectrum of automation in the brain- controlled wheelchairs (BCW) relate to the level of dependence on the human rider to guide the wheelchair, as compared to the wheelchair guiding itself. Many research studies [35] have investigated the inclusion of different operation modes to the brain-controlled wheelchair (BCW). These modes range from low-level (manual), High-level (autonomous), and shared-control (semiautonomous). Below is a detailed explanation for each mode:

- Low-level navigation: where the user is in a complete control of the wheelchair as it directly obeys the user's commands and does not move by itself. The wheelchair is controlled through simple navigation commands, such as "move forward" or "turn left" Also it incorporates basic collision avoidance supports as stopping the wheelchair when obstacles are encountered. Using this navigation mode, users can navigate and perform any path they want, along with having control of the specific movements. In this mode, the system does not assist in the execution of the selected command.

- High-level navigation: where the users have a rough control of the BCW by selecting high-level commands such as "take me to the living room" or "leave this room." The BCW must have some kind of intelligence so that the specific path to the selected destination is transparent to users, i.e., the user does not select specific low- level commands. Also, it can incorporate basic collision avoidance (stopping the wheelchair when obstacles are encountered) and obstacle avoidance (planning a new path to avoid the obstacle) supports.
- Shared-control navigation: where both the user and the system share the control of the BCW. This can be done in two ways: i) users generate lowlevel commands, while the system assists the navigation with features such as obstacle avoidance, or maximum likelihood command execution; and ii) users can switch between a lowand a high-level navigation mode. The user in this mode oversees the navigation and issues highlevel commands while the wheelchair executes the motions. This enables the user to still remain in charge of the decision-making process, but with less involvement in the execution.

\section{RELATED WORK}

Recently, considerable amount of research that tackle the operation modes to the BCW have been reported in literature. In the following we report the work that has been done categorized based on the different modes of navigation:

\section{A. Low-Level Control (Manual)}

The authors in [10] designed a manual BCI-based wheelchair that can be steered by only the users' brain signals. The designed system utilizes three mental tasks that are turning left, right, and go forward. These commands were achieved through the left and right motor imageries to turn left and turn right, respectively, and feet motor imagery to go forward. Similarly, the authors in [11] developed a manually BCI controlled wheelchair using an onboard computer that is responsible for processing and classifying the captured EEG signals to generate wheelchair steering commands. The onboard computer sends through a serial port the generated commands to the wheelchair's motor drivers. The wheelchair can either move forward, turns left or right, or stops.

On the other hand, Li et al. in [12] presented a hybrid brain/muscle interface to manually control a wheelchair. The authors argue that BCI based on P300 or SSVEP can cause the fatigue and dryness of the eye, and then lead to user's inattention. The developed system distinguishes four user's commands, go forward, turning right, turning left, and stop. These commands are captured from the: (1) two mental states corresponding to the wheelchair's motion of turning left and turning right, (2) the EMG signal captured from the user's gritting his/her left teeth and right teeth that correspond to the motion go forward and stop respectfully. The developed wheelchair is equipped with various types of sensors to perceive the environment's context. These sensors are vision camera, and a laser rangefinder (sonar).

The authors in [13], also, developed a hybrid BCI system that combines two types of BCI paradigms (P300 potential and SSVEP) to improve the performance of asynchronous control to instantly and accurately distinguish the control and idle states needed to steer the wheelchair. And considering that the system controls the wheelchair manually, the authors designed the graphical user interface (GUI) to display four groups of buttons, and each group has one large button in the center and eight small buttons surrounding it. When the user concentrates on one group of buttons, both P300 potential and SSVEP can be evoked al the same time. The flickering buttons in each 
group invoke SSVEP, and the flashing of the four large buttons evoke P300 potential. In order for the authors to produce a go or stop commands in wheelchair control this method was used.

Moreover, in [14] Diez et al. developed a wheelchair that can be controlled through a BCI based SSVEP signals. The designed system can discriminate five classes: top, bottom, left, right and undefined. The detected stimuli can be translated to the first four classes, while the undefined class is chosen when no stimuli are detected. The wheelchair moves manually based on the identified class. For example, it moves forward if the BCI detected the top stimulus, turns left if the left stimulus was detected, turns right if the right stimulus was detected, and finally stops if the bottom stimulus was detected. Moreover, when no stimulus was detected (undefined class) the wheelchair, for safety reasons, stops as well. The system provides the user with online feedback indicating the detected stimulus by translating it to its proper steering direction using a blue arrow on the screen. And in the case of an undefined class, a red circle is shown in the center of the screen.

The authors in [15] developed a BCI based electric wheelchair control system. The proposed wheelchair enabled users to steer the wheelchair in four directions forward, backward, turn left or right by utilizing the eyes closing signal for more than one second and without any pre-training. Users wore an EEG acquisition cap that has four lights corresponding to the four directions. These light flashes in a clockwise loop and lasts for a fixed period of time. Moreover, when the user wants to select a specific direction, he/she closes their eyes as soon as the desired direction flashes.

Moreover, the authors in [16] built upon their previous work of [17] where they combined two types of BCI paradigms (motor imagery and P300 potentials) and controlled the wheelchairs direction and speed. The direction control was achieved through two commands (turning left and right), and the speed control was done by controlling the acceleration and deceleration. The authors' new extension combined motor imagery, P300 potentials, and eye blinking to achieve forward, backward, and stop control of a wheelchair respectively. Their work combined with their previous resulted in having the users choose and navigate from seven steering commands.

Cao et al. in [18] developed a hybrid BCI system that combines two BCI paradigms (motor imagery and SSVEP) to concurrently control the speed and direction of a wheelchair. The proposed system manually steers the wheelchair by providing eight commands for the users choose and navigate from. These commands are turn left, turn right, drive forward, accelerate, decelerate, drive at the uniform velocity, and turn on and off the switch. Similarly, the authors in [19] argue that their proposed system allows the user to implement different types of commands in parallel. The proposed combine SSVEP and MI tasks to develop a new hybrid BCI method. It utilizes two-class MI and four-class SSVEP tasks, in which the user imagines moving his/her left or right-hand and focuses on one of four oscillating visual stimuli simultaneously.

In [20], the authors developed a manual control wheelchair navigation system based on a hidden Markov model (HMM). The developed system steers the wheelchair by capturing the electrooculography (EOG) signal originating from the user's eyeball and eyelid movements. A feature extraction was used to determine whether the eyes are open or closed and whether the eyes are gazing to the left, right, or center. These features are used as inputs to the HMM which generates commands for navigating the wheelchair accordingly. The wheelchair is equipped with a proximity sensor to avoid obstacles and it can move forward and backward in three directions.

Varona-Moya et al. in [21] enhanced an electric wheelchair, by incorporating multiple sensors and emulating its analog 2-axis joystick with a custom-built control board. The enhanced system receives the BCI navigation commands through a TCP connection and then transforms them into lowlevel movement commands that are fed to the wheelchair. Moreover, a real-time map of the area surrounding the wheelchair was created using the incorporated eleven ultrasonic rangefinders (sonar). Updating the grid-map in real time was achieved using a sonar model, which upon the detection of an obstacle at a given distance; it updates all the grid cells within the obstacle detected vertex.

In [22] Đumić and Kevrić developed a manually controlled wheelchair. The user controls the direction of the wheelchair through a BCI headset that detect an eye blinking action. There are four directions the user can choose from in order to steer the wheelchair, which are: left, right, forward, and backwards. The actual wheelchair was enhanced by using a microcontroller to control the joystick by servo motors.

\section{B. High-Level Control (Autonomous)}

Define The authors in [23] designed a brain-controlled wheelchair, which interacts with the user using a simple interface. The navigation system proposes a semantic map that integrates the navigation points, semantic targets, and a local 3D map. The semantic targets provide the recognized objects' type, outline and its functionality information, for example, if the object was identified to be a table, then its associated information is that it can be docked. The local 3D map provides a traversable navigation point. The user chooses one of the navigation points from the semantic map as the destination goal using a brain-computer interface (BCI).

In [24], Ng et al. developed a BCI controlled wheelchair based on the steady-state visual evoked potential (SSVEP) paradigm. The proposed system takes the desired destination from the user through a BCI signal and communicates it to the wheelchair navigation system to plan a path autonomously while avoiding obstacles on the way to the destination. The responsibility of controlling the wheelchair is switched from the user to the navigation software, which reduces the number of BCI commands needed to steer the wheelchair to the desired destination.

Zhang et al. in [25] developed an autonomous wheelchair where the user selects a destination from the map using one of the BCI paradigms (motor imagery or P300). Based on the selected destination, the navigation component plans a short path and steers the wheelchair to the desired goal. Furthermore, the user can choose to stop the wheelchair by issuing a stop command using the BCI whenever he/she wants. The authors claim that their system reduces the user's mental burden substantially. 


\section{Shared Control (Semi-autonomous)}

The authors in [26] introduced a shared control architecture that combines the user's intention along with the precision of a powered wheelchair. Their system combines BCI with a shared control architecture that permits users to produce dynamic and simple navigation directions, as opposed to users being seated and relying on a predefined path for most of the navigation time. The shared controller decides what actions ought to be taken, based on the user's input (turning left or right) while taking into account the context of the environment, which was perceived using ten sonar sensors and two webcams. The proposed system was evaluated against four healthy experienced BCI users.

Lopes et al. in [27] propose a robotic assistive navigation wheelchair that integrates the Brain-Computer Interface (BCI) technology, as the Human- Machine Interface (HMI). In their paper, the authors proposed a two-layer collaborative control approach that takes into account both the user and the machine commands to guide and maneuver the wheelchair. The first layer in their proposed approach is a virtual-constraint layer. It is responsible for enabling/disabling the user commands, based on the environment's context. For example, user commands are enabled in the situation of multiple directions that was caused by newfound obstacles in the environment. The second layer is responsible for matching the user's intended BCI commands to a suitable steering command, considering the client capability to control the wheelchair and situation awareness of potential directions at a given location.

Moreover, in [28] the authors proposed a new sharedcontrol approach for a brain- controlled wheelchair. The shared controller switches between two controlling agents, the BCI control agent, and an autonomous control agent taking into consideration both the context of the environment and the intention of the user. The architecture of the proposed approach consists of four layers: the human- machine interface, the global motion planning, the local motion planning, and the motion control. The global motion planning layer comprises of the knowledge database that was built by the SLAM method to store information about the location of obstacles as well as the kinematics of the wheelchair. This layer receives an output from the BCI and determines the path to the goal by using the NRPF algorithm. It also calculates the distances between the wheelchair and the goal as well as the distances between the wheelchair and the obstacle. Moreover, the distance to the obstacle decides the mode of control. For example, the autonomous control agent is activated once an obstacle is detected using a laser scanner, which results in enabling the local path-planning module to return a collision-free path by employing an improved potential field method. Finally, the motion control layer receives direction commands from the local motion-planning layer. The received commands determine the movement of the wheelchair.

Chen et al. in [29] proposed a hybrid BCI scheme based on brain electrophysiology (EEG) signals, a shared control system of a bionic manipulator is designed and a motion planning of the wheelchair. The wheelchair is equipped with an obstacle avoidance system that is composed of 8 photoelectric sensors distributed across the wheelchair. The proposed system is comprised of the human-robot interface and EEG signals that are used to identify the steering command expressed by the user's motion imagery, which is achieved by imaging motions of the left and right legs, left and right hands and so on. The user's expressed commands are translated into the corresponding control actions, which are sent to the actuator of the arm joint motor to realize the motion control of the bionic manipulator.

Moreover, researchers in the field have used a number of different metrics to evaluate their proposed the BCW. However, there are a limited number of research that employ the usability evaluation metrics. For example, from the reviewed literature only [20] and [14] evaluated their BCW using usability, learnability, and measured the user's experience. The rest of the reviewed papers evaluated their BCW in terms of how accurate their system completed the task and the time it took among other factors.

Furthermore, prior to the test session, most studies performed a training phase to reduce the effect of unfamiliarity to the technology used. The orientation session aims to introduce the participants to the subject in general and to the system in particular. Some studies considered the users' familiarity with the test BCI technology. Besides, the profile and number of test participants varied among the studies: Lopes et al. [27] recruited 11 participants one of which has a cerebral palsy and motor impairment, Diez et al. [14] recruited 13 participants, with one being a paraplegic participant. While another study in [24] evaluated the system against 37 healthy participant. Other studies in [10]-[13], [15], [16], [18], [21][23], [25], [26], [28], [29] recruited lesser number of test participants ranging between one to eight participants.

In summary, the problem of choosing one signal or another depends on a number of factors like presence or absence of a graphical user interface (GUI), the number of commands, and the need to implement a continuous control of the wheelchair. The P300 requires a GUI to be present and has a discrete control mechanism as opposed to the ERD/ERS. Furthermore, we noticed that a few of the reviewed research handled the semi- autonomous (shared level) navigation and combining it with obstacle detection and employing a path planning algorithm to find the shortest path to the goal. Remarkably, most the research (63.16\% of the papers) uses a manual (low level) navigation system without adding an obstacle detection and avoidance mechanism. On the other hand, the P300 used mostly high-level navigation commands.

Moreover, some of the reviewed high-level BCWs focus solely on the BCI interface without mentioning the path planning technique used. Similarly, some works like [23], [24], and [25] did not consider applying an obstacle detection and avoidance technique, this might be attributed to the fact that they are building their system to navigate in a static environment without taking into consideration the changes that might suddenly occur in the environment.

It should be noted that the evaluation criteria used in the different studies were heterogeneous, where no standard evaluation metrics were followed. When taking the type of signals into account the BCW that were based on the ERD/ERS or SSVEP paradigms all gave a great importance to the evaluation of accuracy in selection the commands, along 
with the time it was required to complete the path. On the other hand, half of the BCW adapting the P300 paradigm evaluated the selection time, which is defined as the time it takes the user to select the desired command. However, the selection time cannot be used when evaluating a BCW that is based on ERD/ERS signals, due to the difficulty of exactly knowing when the user starts the selection process.

This paper extends this line of research and proposes a software framework integrating the three different operating modes. A prototype system based on this framework was implemented including three components: the navigation and path planning, the obstacle detection, and the user interface. Finally, the usability of the proposed system was evaluated against standardized evaluation metrics.

\section{Proposed Framework Design ANd Architecture}

This paper proposes to design and develop a software framework for a BCI controlled wheelchair that gives the user the ability to choose one of the three navigation methods: manual/direct control (low level control), semi- autonomous (shared control), and autonomous (high level control).

In the manual/direct (low level control), the user controls the wheelchair by only his thoughts. In the semi-autonomous (shared control), the control takes the user's intention into account while doing the planning and the wheelchair follows the orders feed to it from a planner. The user, however, might express his/her intention to control the wheelchair and steer it by merely using the BCI. When the user's steering intention conflicts with the planner's instructions, the control system will replan the task. Finally, in the autonomous (high level control) the user will only have to select the destination and let the system plan a safe path to the destination while avoiding any obstacles on the way. However, in all the three navigation methods, the wheelchair will be equipped with obstacle detection sensors in order to detect and avoid the obstacle.

\section{A. High-level Framework Architecture}

As a typical navigation system, the framework consists of four main components: the indoor positioner component, the navigator component, the obstacle detection component, and the user interface component. The architecture of the whole system is represented in FIGURE 1.

1) Positioning component: The indoor positioner component gathers relevant information concerning the navigated environment and the user's current position and makes this information available for the other component to use. These components include the navigation component that generates the navigational directions based on the positioner information and the interface component that outputs related contextual information based on the positioner information.

2) Navigation component: Navigation including path planning is a key component to any navigational system, since it is responsible for computing the optimal path to reach the destination selected by the user, taking into account the user preferences including the route length, number of turn and safety priorities. The computed path is then translated to commands fed into the wheelchair to guide the user through the environment. Moreover, Path planning environments can either be static or dynamic, and according to them, two different path-planning approaches exist: local and global path planning. Global planning assumes a prior knowledge of the environment, a predefined map and fixed obstacle and then computes the overall path to destination. This approach assumes a static environment and is usually used for indoor navigation [30]. On the other hand, local planning aims to locally plan an obstacles-free path and determine how to navigate around obstacles. The local planning is usually used in robotics since the construction and maintenance of a global map may become computationally complex for a robot. This component also includes the shared controller which receives commands from the user and the machine, evaluates the situation and the current environment and then makes the appropriate decision. This decision is then translated into commands via the Path Translator to control the wheelchair.

3) Obstacle detection and avoidance component: The obstacle detection component, detects the existence of an obstacle, notifies the user and takes the appropriate action, which is identifying the obstacle on the map and then communicats with the navigator component to plan an alternative path to avoid the obstacle in order to reach to the goal destination. It should be noted that the control is passed when an obstacle is detected to so the wheelchair can manuver around the obstacle autonomously.

4) User interface component: Specifying a component for handling the interaction with the user and ensuring an instant response to user's requests is essential for any system. This component gets the preference of the user on how to plan a path to navigate the environment to reach the desired destination and then communicates with the navigation component so that the user's preference and destination point are fed into the path-planning algorithm. Moreover, the user interface component notifies the user when an obstacle is detected.

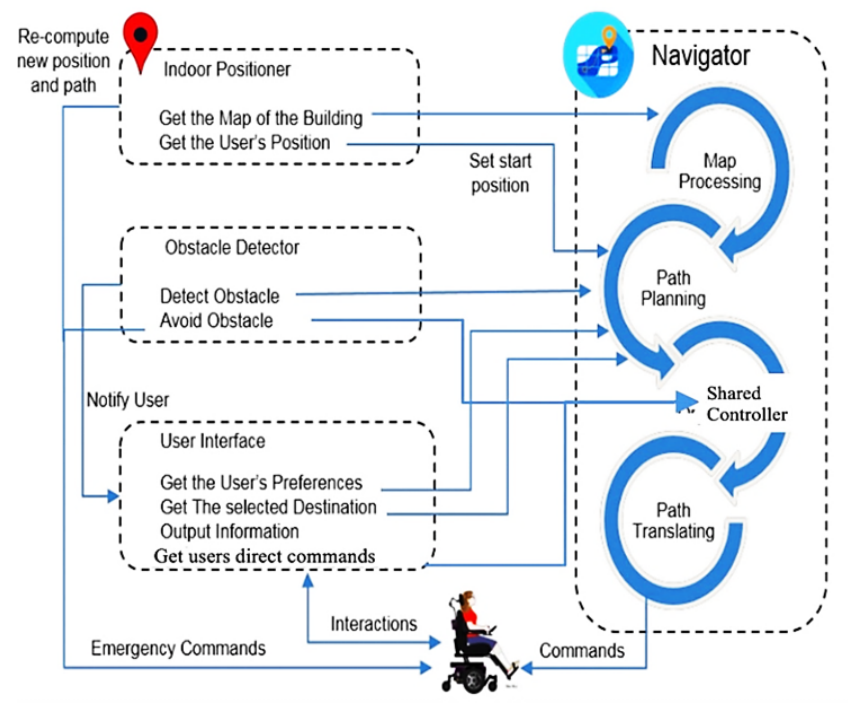

Fig. 1. Wheelchair High-level Framework Architecture. 


\section{B. Shared Control System Architecture}

As the main focus is to study and assess the usability of the shared control navigation, 3 components from the aforementioned components were considered: namely Navigation, Obstacle Detection and User Interface. The architecture of the proposed shared control system was designed in layers to interact synchronously so that each component can communicate independently to send or receive information. The architecture presented in FIGURE 2 is structured in four layers: Human Machine Interface (HMI), Global Motion Planning, Local Motion Planning, and Motion Control.

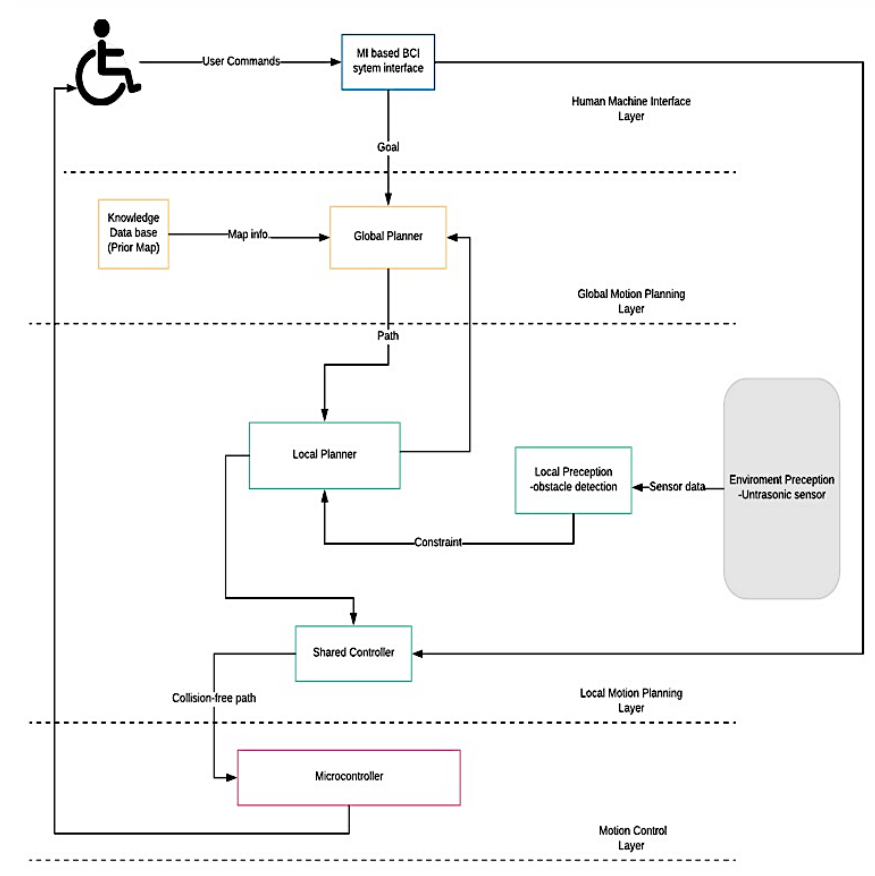

Fig. 2. The Proposed Shared Control System Architecture.

The first layer implements the input interface of the User Interface component. In this layer, an MI-based BCI is used to provide the user intent, which are the issued steering commands. The Navigator component was implemented across three layers. The global planner determines the path to a predefined goal, based on a priori grid map information. The local planner plans a new path to avoid new detected obstacles in the environment. The shared-controller determines the set of appropriate moves to reach a predefined goal combining both the user and the machine commands. The obstacle detection is implemented in the third layer, along with the local planner, and is performed out based on ultrasonic sensor information.

\section{Implemented System Components}

The implemented components include the BCI interface, navigation component and obstacle detection.

\section{- BCI Interface Component:}

For BCI, hybrid BCI that uses both motor imagery and P300 potential approach was adapted in this system. The idea of hybrid BCI is to activate and control the system by imagining performing specified tasks. First, the device has to be trained in order to recognize the brain patterns of a user (motor imagery). Users were trained in two mental commands: neutral, and push. The choice of having only two mental commands rather than associating each task with a command for itself; is due to the user's difficulty of distinguishing different commands and thus increasing his/her burden.

The neutral command is trained by asking the user to stay idle and think of nothing for two seconds, in which the user does not perform any command. And the push command is trained by asking the user to imagine pushing the cube for four seconds. These commands, however, have to be trained multiple times in order for the commands to have a high confidence.

Furthermore, the P300 was used to control which action of direction the wheelchair has to take (right, left, forward, backward). For example, to turn right, the user imagines push movement while focusing on the right arrow flashing button on the graphical user interface (GUI).

\section{- Obstacle Detection Component}

The wheelchair will be equipped with Ultrasonic sensors mounted on its front part in order provide a safety mechanism against collision. If the measured distance to the obstacle is smaller than $50 \mathrm{~cm}$, the map is updated with the new obstacle and the local planner will plan a path to avoid the obstacle.

- Navigation Component

\section{o Vector Map}

A two- dimensional vector map of the environment will be manually constructed and pre- processed to define the obstacles' locations so that the generated path can avoid intersecting with them later. The map will be segmented into a grid of equal sized cells ( 1 meter of each cell). It is assumed that all doors and windows on the wall are to be closed and are considered as normal walls. This assumption implies that the wheelchair cannot travel through them.

\section{o Path Planning}

The proposed system includes two path-planning algorithms: (1) the global path planner finds the shortest path from initial location to the goal, (2) the local path planner to plan a path around the obstacle to avoid it. The goal (endpoint) is assumed to be selected by the user.

The D*Lite, global path planning algorithm is used to move a robot equipped with an ultrasonic sensor for detecting obstacles in order to move from the start point to a goal point assuming that the environment and all obstacles are known, and the size of each cell equals the size of the robot. For the local planning part, a local repair strategy called path splicing was used. The path splicing strategy finds a path nearby and assumes the path farther away need not be recomputed until we get closer to it. So instead of recalculating the entire path, the first $\mathrm{M}$ steps of the path are calculated.

\section{o Shared Controller}

The shared controller receives commands from two agents, the user and the machine. The user issues BCI commands using the BCI headset. While the machine commands are issued from the application. However, instead of directly executing the 
user's commands, the shared control component evaluates the situation first. The current environment, perceived through ultrasonic sensors, is taken into considerations.

The shared controller has two levels of support that are only initiated when the situation calls for them. The two levels of support are collision avoidance and obstacle avoidance that will be activated near obstacles to prevent collisions.

The collision avoidance is thought of as an emergency stop. For example, when the user moves the wheelchair too close to an obstacle, the velocity will be decreased until it comes to a full stop. The ultrasonic sensors, mounted on the wheelchair, are used to determine when to activate this behavior. The activation threshold was set at $0.4 \mathrm{~m}$ to maintain the safety of the user. If the ultrasonic sensors detect obstacles within this threshold, the collision avoidance behavior will be activated. However, unlike the previous behavior, the obstacle avoidance employed the use of the local planning strategy in order to steer the robot away from the obstacle. This behavior takes both the input of the user and the environment into consideration, to properly assist the local planning strategy.

\section{Deployment And Navigation Modes}

The proposed system consists of three main nodes. The first node is the Emotiv insight headset to capture EEG signal from the user. The second node hosts the software application that receives the captured EEG signal from the headset and converts it into commands. It also plans an obstacles free path to the destination. The third node is the microcontroller to control the wheelchair movement. However, due to limited funding, the wheelchair could not be acquired and was replaced by a prototyped robot. The robot is a Boe-Bot robot equipped with an Arduino UNO as the microcontroller to operate two motors and several ultrasonic sensors.

When using the Emotiv insight headset, the user was seated and asked to focus his attention to the command he wished to instruct. It is important to note that to know that every person has unique EEG signals. Therefore, every participant has to go through a training session before proceeding with the system. Furthermore, the system's nodes are able to share messages between them via Bluetooth. The software application component can control the robot through sending commands as a string of characters. The commands used in the system are: forward (F), turn 90 degree to the left (L), turn 90 degree to the right $(\mathrm{R})$, and stop $(\mathrm{S})$.

\section{Pilot Usability Evaluation}

The (ISO 9241-11: 2018) identifies effectiveness, efficiency and satisfaction as major attributes of the usability. To evaluate the usability of the proposed system, test tasks were designed to assess the metrics for measuring the required criteria. Hence, the effectiveness was evaluated in terms of task completion, the efficiency was measured based on completion time and workload, and the satisfaction was assessed using the System Usability Scale (SUS).

\section{A. Usability Evaluation Materials}

A prototype based on a car robot (Boe-Bot) was used to simulate the wheelchair. The robot car is equipped with an ultrasonic sensor to detect obstacles. The assembled robot is shown in FIGURE 3.

1) Moreover, the Emotiv Insight [33] was used as the BCI headset to read and transmit EEG signals. Additionally, the BCI interface has been designed as a visual oddball paradigm. The paradigm comprises four steering commands, encoded by the following symbols: FORWARD, RIGHT90, LEFT90 and STOP, as shown in FIGURE 4. These symbols flash randomly. At a given moment, the relevant steering event is the symbol mentally selected by the user, which corresponds to the direction he/she wants to follow, and all other flashing symbols are discarded and considered as a non-relevantevent.

The map for room used for the evaluation is shown in FIGURE 5. The room is an $8 \times 8 \mathrm{~m}^{2}$, where the green circle is the starting point and the purple circle is the final destination (goal).

\section{B. Usability Evaluation Method}

Each experimental session was designed to last for a duration of about one hour, during which subjects were asked to control the robot using the Emotiv headset. A detailed flow is presented in Figure 6.

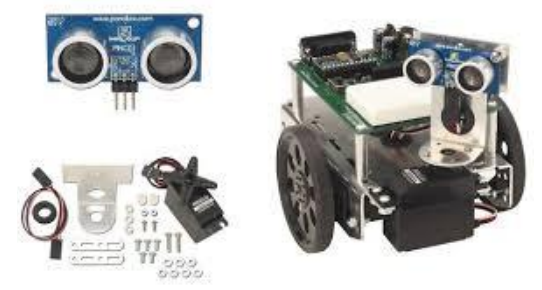

Fig. 3. The Assembled Robot.

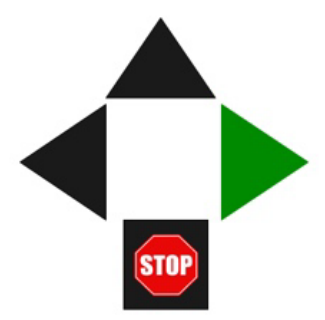

Fig. 4. The P300 GUI.

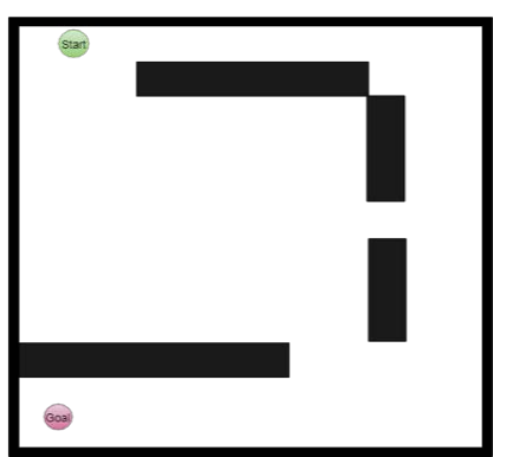

Fig. 5. Experimental Room Mock-up. 


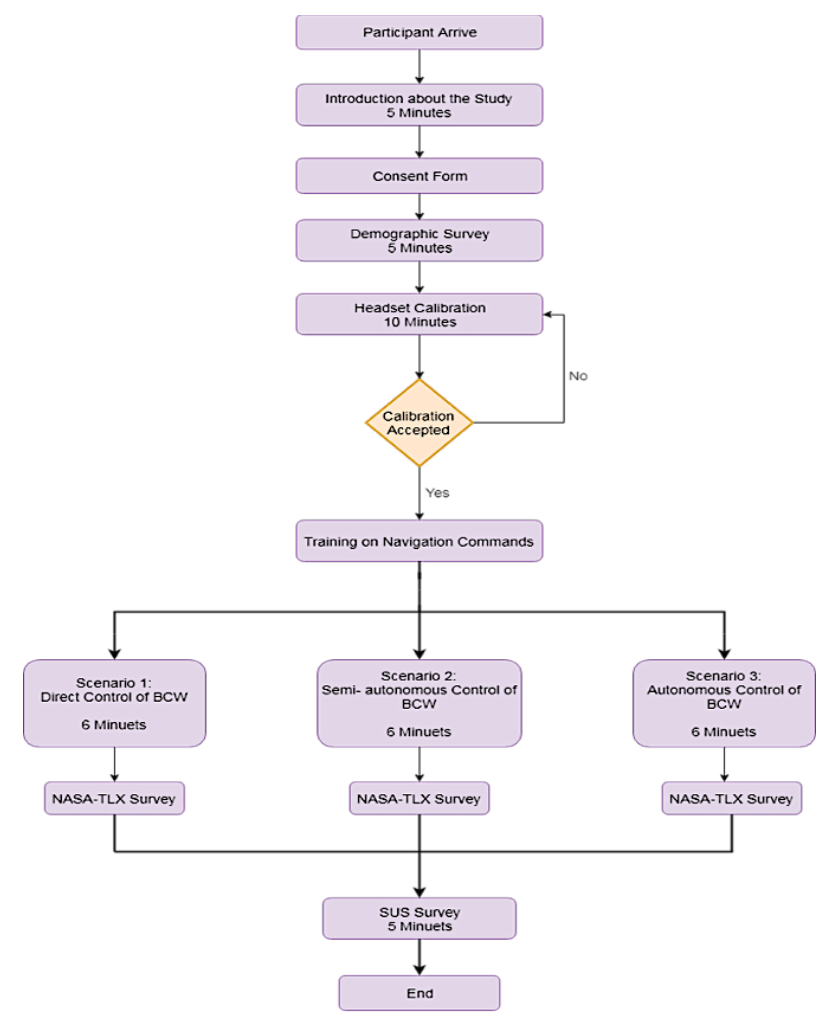

Fig. 6. The Experimental Session Procedure.

Each session began with asking the participant to fill out a demographic questionnaire and sign a consent form. Then, the Emotiv headset was mounted on the participant for calibration. Calibration involves mounting the headset on the participant and ensuring a good signal for each of the five electrodes (green color). In some cases, a fair signal (yellow color) is accepted. Following the calibration and before the online session, each participant was asked to perform an off-line training to control wheelchair, this training was done until the participant's signal recognition accuracy reaches above 85 percent.

After the calibration and off-line training, the participants were asked to perform three real-time navigation scenarios (direct control, semi-autonomous, and autonomous control) for a duration of six minutes each. The navigation scenarios all took place in a structured known environment that included both static mapped obstacles and an obstacle/s new to the environment.

The first navigation scenario, denoted by scenario 1 , was to directly control the wheelchair. The participant is instructed to focus on the GUI that has four symbols flash randomly. These symbols correspond to the four steering commands (forward, turn right 90 degree, turn left 90 degree, and stop). When the participant chooses to go forward the robot continues performing this command till the users issues another command or the robot stops due to facing an obstacle. Moreover, in the second navigation scenario, denoted by scenario 2, where the participants share the responsibility of controlling the wheelchair with system. The wheelchair autonomously moves from the starting point to the destination while avoiding obstacles. However, the participants can interfere anytime and change the direction of the wheelchair and steer it as he/she wishes. Once the participant is done with commanding the wheelchair, the system takes back the control and navigates the wheelchair back to the destination. Finally, in the third navigation scenario, denoted by scenario 3. The participant is instructed to sit still while the wheelchair is navigating the environment to reach to the final destination (goal) while avoid the new introduced obstacle. It is also worth noting that the order of the three navigation scenarios was counter-balanced across the participants as shown in TABLE 1

TABLE I. THE SEQUENCE OF THE 3 NAVIGATION SCENARIOS FOR EACH PARTICIPANT

\begin{tabular}{|l|l|}
\hline ID & Sequence of Navigation Scenarios \\
\hline P1 & Scenarios 1, 2, 3 \\
\hline P2 & Scenarios 1, 3, 2 \\
\hline P3 & Scenarios 2, 1, 3 \\
\hline P4 & Scenarios 3, 2, 1 \\
\hline P5 & Scenarios 2, 3, 1 \\
\hline
\end{tabular}

\section{Evaluation Participants}

TABLE 2 illustrates the demographics of the participants taking part in the usability evaluation. The participants were mainly recruited through the use of social networks and word of mouth. All the participants took part in an initial calibration task. This initial calibration was required in order to have an acceptable accuracy of the acquired brain signals, which would enable the participants to control the BCW in the navigation task.

TABLE II. The DEMOgRaphics OF THE PARTICIPANTS TAKING PART IN THE USABILITY EVALUATION

\begin{tabular}{|l|l|l|l|l|}
\hline ID & Gender & Age & $\begin{array}{l}\text { BCI } \\
\text { Experience }\end{array}$ & $\begin{array}{l}\text { Degree of Motor } \\
\text { Disability }\end{array}$ \\
\hline P1 & Female & 29 & No & None \\
\hline P2 & Male & 22 & No & None \\
\hline P3 & Male & 24 & No & Fractured leg \\
\hline P4 & Female & 66 & Yes & None \\
\hline P5 & Female & 25 & No & None \\
\hline
\end{tabular}

\section{Evaluation Results}

The participants were asked to perform the navigation scenario they are presented with. The scenario is considered completed if the participant performs the navigation in less than 6 minutes. Moreover, all the participants have completed the navigation scenarios in a short time period for the direct control scenario (scenario 1) except P1. These results range from 4.9 to 6.2 minutes. It can be seen that P4 completed the scenario in short time which is attributed to the fact that the participant has prior knowledge on how to use the BCI. However, P1 got the highest completion time, which indicates that the user faced some difficulty using the BCI.

Furthermore, it can be seen from FIGURE 7 that the participants' completion time improved and this can be attributed to the learning effect. In addition, it was observed that the sequence of the navigation scenarios that the 
participants were supposed to follow affected the completion time. P1 and P2 for example took scenario 1 which is the direct control first and that helped them get familiar with how BCW work and got a first-hand experience on how to control the prototype via a sequence of commands. Also, according to the evaluation model this would result in a high efficiency of the proposed framework.

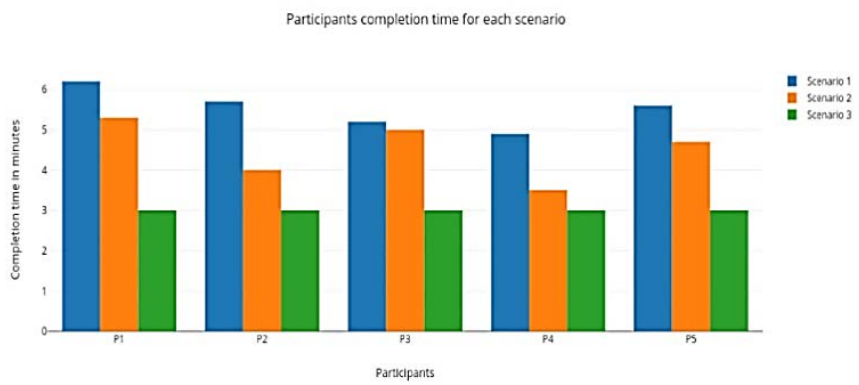

Fig. 7. Navigation Completion Time Periods among Participants for different Three Scenarios.

Moreover, the overall results from the NASA-TLX [34] workload survey showed that the participants' perceived the BCW as having an average physical demand, although, the BCW experiments does not require movement. Therefore, we believe this average score in physical demand was due to fatigue from sitting during the set-up and calibration period, and the increased test length. We believe the increase in mental demand and effort in scenario 1 and 2 was because the BCW experiments required people to focus their attention on making selections, compared to scenario 3 where the participants sat still and let the system do the steering. Nevertheless, it should be noted that in the autonomous navigation, participants reported they felt some frustration, which could be attributed to the fact that the participants might have wanted some navigation control over the prototyped BCW.

The overall analysis reported in FIGURE 8 shows that the workload perceptions increased significantly when the participants were dealing with scenario 1 , which is when the workload demand increased.

Moreover, regarding the accuracy in selection the correct command. It was noted that previous experience in using the BCI can affect the accuracy results. P4, which had a previous experience in using a BCI application performed better and had a high accuracy in selecting the right command. However, with more training it is expected that the other participants can perform the same and improve their selection accuracy.

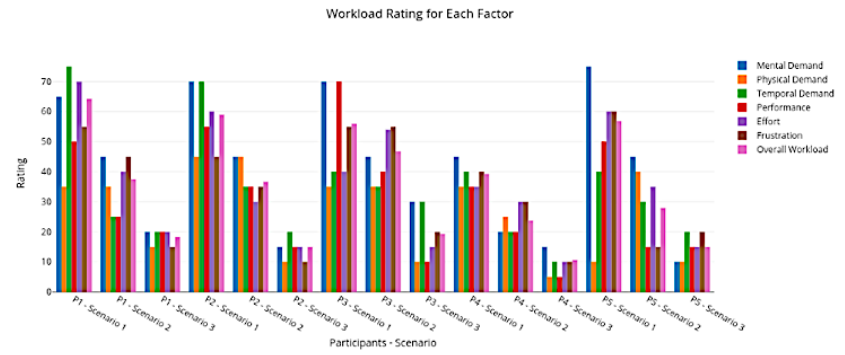

Fig. 8. Workload Rating for each Factor for each Participant/Scenario.
Additionally, according to the SUS questionnaire results, in that were filled by the participants after testing the system to measure the likability of the solution provided. It was found that participants' opinions were diverse in regard to their experience of using the prototyped BCW. While one participant found it difficult to use the system, two found the experience relatively easy and two were neutral in this regard. This applies to their view of the system and whether it can be described as an easy-to-use system or not, despite their own experience with it. Three of the participants believe that they don't require any specialized help in order to be able to use the system. Only two of the participants think that training on the system is required before using it.

Moreover, the majority of the participants agreed the stimuli were easy to distinguish and understand. Moreover, steering the prototyped BCW in an autonomous navigation mod was considered to be easy, similarly, steering the prototyped BCW in a semi- autonomous mode. On the other hand, three of the participants found that steering the prototyped in a direct control mode was difficult. Overall, the participants showed an interest in using the BCW once it becomes available in the market.

Table 3 and Figure 9, that were filled by the participants after testing the system to measure the likability of the solution provided. It was found that participants' opinions were diverse in regard to their experience of using the prototyped BCW. While one participant found it difficult to use the system, two found the experience relatively easy and two were neutral in this regard. This applies to their view of the system and whether it can be described as an easy-to-use system or not, despite their own experience with it. Three of the participants believe that they don't require any specialized help in order to be able to use the system. Only two of the participants think that training on the system is required before using it.

Moreover, the majority of the participants agreed the stimuli were easy to distinguish and understand. Moreover, steering the prototyped BCW in an autonomous navigation mod was considered to be easy, similarly, steering the prototyped BCW in a semi- autonomous mode. On the other hand, three of the participants found that steering the prototyped in a direct control mode was difficult. Overall, the participants showed an interest in using the BCW once it becomes available in the market.

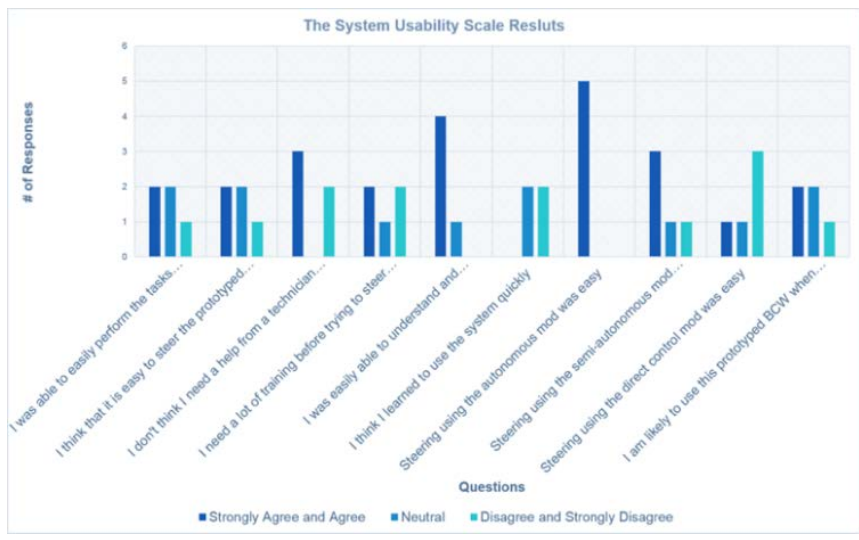

Fig. 9. System Usability Scale (SUS) Results. 
TABLE III. SUS REsUlts (1: STRONGLY AgREE, 5: StRONGLy DisAgREE)

\begin{tabular}{|l|l|l|l|l|l|l|}
\hline Question & $\mathbf{1}$ & $\mathbf{2}$ & $\mathbf{3}$ & $\mathbf{4}$ & $\mathbf{5}$ & $\begin{array}{l}\text { Mean } \\
\text { Opinion } \\
\text { Score }\end{array}$ \\
\hline $\begin{array}{l}\text { I was able to easily perform the } \\
\text { tasks required using the BCI }\end{array}$ & 0 & 2 & 2 & 1 & 0 & 2.2 \\
\hline $\begin{array}{l}\text { I think that it is easy to steer the } \\
\text { prototyped BCW }\end{array}$ & 1 & 1 & 2 & 1 & 0 & 2.6 \\
\hline $\begin{array}{l}\text { I think that I need a help from a } \\
\text { technician person in order to be } \\
\text { able to steer the prototyped BCW }\end{array}$ & 1 & 1 & 0 & 1 & 2 & 3.4 \\
\hline $\begin{array}{l}\text { I need a lot of training before } \\
\text { trying to steer the prototyped } \\
\text { BCW }\end{array}$ & 0 & 2 & 1 & 2 & 0 & 3 \\
\hline $\begin{array}{l}\text { I was easily able to understand } \\
\text { and distinguish the application's } \\
\text { stimuli }\end{array}$ & 2 & 2 & 1 & 0 & 0 & 1.8 \\
\hline $\begin{array}{l}\text { I think I learned to use the system } \\
\text { quickly }\end{array}$ & 0 & 0 & 2 & 2 & 1 & 3.8 \\
\hline $\begin{array}{l}\text { Steering using the autonomous } \\
\text { mod was easy }\end{array}$ & 3 & 2 & 0 & 0 & 0 & 1.4 \\
\hline $\begin{array}{l}\text { Steering using the semi- } \\
\text { autonomous mod was easy }\end{array}$ & 3 & 1 & 1 & 0 & 0 & 1.6 \\
\hline $\begin{array}{l}\text { Steering using the direct control } \\
\text { mod was easy }\end{array}$ & 1 & 1 & 2 & 1 & 3.6 \\
\hline $\begin{array}{l}\text { I am likely to use this prototyped } \\
\text { BCW when it becomes available } \\
\text { in the apps market }\end{array}$ & 2 & 2 & 1 & 0 & 2.8 \\
\hline
\end{tabular}

\section{CONCLUSION}

The work of this research was motivated by number of factors in order to improve the quality of life for individuals with mobility impairments since most of the smart Wheelchairs developed have hardware and software architectures that are specific for the wheelchair model developed and are usually very difficult to configure in order for the physically impaired individuals to start using them.

In this work, we proposed a framework by engineering three components (navigation and path planning, obstacle detection, and user interface) taking into consideration the usability and safety requirements to develop a brain- controlled wheelchair for mobility-impaired individuals to help them navigate their way seamlessly in an indoor environment. However, number of issues were encountered resulting in number of limitations. Among these limitations was that the framework was developed using a prototype rather than a real wheelchair, which is attributed to the limited and late funding. On the usability aspect, the small number and lack of variance within the evaluation sample might, though it is considered as an initial evaluation.

In the future we aim to test the proposed framework with a real wheelchair in addition to increasing the number and variance of the evaluation sample.

\section{ACKNOWLEDGMENT}

The authors acknowledge the financial support provided by King Abdulaziz City for Science and Technology (KACST) by grant number (1-17-02-001-0031).

\section{REFERENCES}

[1] J R. C. Simpson, "Smart wheelchairs: A literature review," J. Rehabil. Res. Dev., vol. 42, no. 4, p. 423, 2005.

[2] N. E. Klepeis et al., "The National Human Activity Pattern Survey (NHAPS): a resource for assessing exposure to environmental pollutants," J. Expo. Anal. Environ. Epidemiol., vol. 11, no. 3, pp. 231252, Jun. 2001.

[3] R. Simpson et al., "A prototype power assist wheelchair that provides for obstacle detection and avoidance for those with visual impairments," Journal of neuroengineering and rehabilitation, vol. 2, p. 30, Nov. 2005, doi: 10.1186/1743-0003-2-30.

[4] R. A. M. Braga, M. Petry, A. P. Moreira, and L. P. Reis, "Concept and Design of the Intellwheels Platform for Developing Intelligent Wheelchairs," in Informatics in Control, Automation and Robotics: Selcted Papers from the International Conference on Informatics in Control, Automation and Robotics 2008, J. A. Cetto, J.-L. Ferrier, and J. Filipe, Eds. Berlin, Heidelberg: Springer Berlin Heidelberg, 2009, pp. 191-203.

[5] "Impaired Physical Mobility - Nursing Diagnosis \& Care Plan Nurseslabs.” [Online]. Available: https://nurseslabs.com/impairedphysical-mobility/. [Accessed: 10-Oct-2017].

[6] "What is assistive technology?" [Online]. Available: https://www.washington.edu/accessit/print.html?ID=1109. [Accessed: 10-Oct-2017].

[7] P. C. Garrido, I. L. Ruiz, and M. Á. Gómez-Nieto, "Support for Visually Impaired through Mobile and NFC Technology," in IT Revolutions, 2011, pp. 116-126.

[8] D. E. Hinton Sr, "Research and technological aids for people who are deaf-blind,” Am. Rehabil., vol. 15, no. 2, pp. 7-11, 1989.

[9] W. Hasselbring, "Component-based software engineering," Handb. Softw. Eng. Knowl. Eng., vol. 2, pp. 289-305, 2002.

[10] J. Li, J. Liang, Q. Zhao, J. Li, K. Hong, and L. Zhang, "DESIGN OF ASSISTIVE WHEELCHAIR SYSTEM DIRECTLY STEERED BY HUMAN THOUGHTS,” Int. J. Neural Syst., vol. 23, no. 03, p. 1350013, Jun. 2013.

[11] S. M. T. Müller, T. F. Bastos, and M. S. Filho, "Proposal of a SSVEPBCI to Command a Robotic Wheelchair,” J. Control Autom. Electr. Syst., vol. 24, no. 1, pp. 97-105, Apr. 2013.

[12] Z. Li, S. Lei, C. Su, and G. Li, "Hybrid brain/muscle-actuated control of an intelligent wheelchair," in 2013 IEEE International Conference on Robotics and Biomimetics (ROBIO), 2013, pp. 19-25.

[13] Y. Li, J. Pan, F. Wang, and Z. Yu, "A hybrid BCI system combining P300 and SSVEP and its application to wheelchair control," IEEE Trans. Biomed. Eng., vol. 60, no. 11, pp. 3156-3166, 2013.

[14] P. F. Diez et al., "Commanding a robotic wheelchair with a highfrequency steady-state visual evoked potential based brain-computer interface,” Med. Eng. Phys., vol. 35, no. 8, pp. 1155-1164, Aug. 2013.

[15] D. Ming et al., "Electric wheelchair control system using brain-computer interface based on alpha-wave blocking,” Trans. Tianjin Univ., vol. 20, no. 5, pp. 358-363, Oct. 2014.

[16] H. Wang, Y. Li, J. Long, T. Yu, and Z. Gu, “An asynchronous wheelchair control by hybrid EEG-EOG brain-computer interface,” Cogn. Neurodyn., vol. 8, no. 5, pp. 399-409, Oct. 2014.

[17] J. Long, Y. Li, H. Wang, T. Yu, and J. Pan, "Control of a simulated wheelchair based on a hybrid brain computer interface," Conf. Proc. Annu. Int. Conf. IEEE Eng. Med. Biol. Soc. IEEE Eng. Med. Biol. Soc. Annu. Conf., vol. 2012, pp. 6727-6730, 2012.

[18] L. Cao, J. Li, H. Ji, and C. Jiang, "A hybrid brain computer interface system based on the neurophysiological protocol and brain-actuated switch for wheelchair control,” J. Neurosci. Methods, vol. 229, pp. 3343, May 2014.

[19] J. Li et al., "Evaluation and application of a hybrid brain computer interface for real wheelchair parallel control with multi-degree of freedom,” Int. J. Neural Syst., vol. 24, no. 4, p. 1450014, Jun. 2014.

[20] F. Aziz, H. Arof, N. Mokhtar, and M. Mubin, "HMM based automated wheelchair navigation using EOG traces in EEG,” J. Neural Eng., vol. 11, no. 5, p. 056018, 2014. 
[21] S. Varona-Moya, F. Velasco-Álvarez, S. Sancha-Ros, Á. FernándezRodríguez, M. J. Blanca, and R. Ron-Angevin, "Wheelchair navigation with an audio-cued, two-class motor imagery-based brain-computer interface system," in 2015 7th International IEEE/EMBS Conference on Neural Engineering (NER), 2015, pp. 174-177.

[22] D. Đumić and J. Kevrić, "BCIs for Electric Wheelchair," in Advanced Technologies, Systems, and Applications II, 2018, pp. 833-847.

[23] Z. Wei, W. Chen, J. Wang, H. Wang, and K. Li, "Semantic Mapping for Safe and Comfortable Navigation of a Brain-Controlled Wheelchair," in Intelligent Robotics and Applications, 2013, pp. 307-317.

[24] D. W. Ng, Y. Soh, and S. Goh, "Development of an Autonomous BCI Wheelchair," in 2014 IEEE Symposium on Computational Intelligence in Brain Computer Interfaces (CIBCI), 2014, pp. 1-4.

[25] R. Zhang et al., "Control of a Wheelchair in an Indoor Environment Based on a Brain-Computer Interface and Automated Navigation,” IEEE Trans. Neural Syst. Rehabil. Eng., vol. 24, no. 1, pp. 128-139, Jan. 2016.

[26] T. Carlson and J. del R. Millan, "Brain-Controlled Wheelchairs: A Robotic Architecture,” IEEE Robot. Autom. Mag., vol. 20, no. 1, pp. 6573, Mar. 2013.

[27] A. C. Lopes, G. Pires, and U. Nunes, "Assisted navigation for a brainactuated intelligent wheelchair,” Robot. Auton. Syst., vol. 61, no. 3, pp. 245-258, Mar. 2013.

[28] J. Duan, Z. Li, C. Yang, and P. Xu, "Shared control of a brain-actuated intelligent wheelchair," in Intelligent Control and Automation (WCICA), 2014 11th World Congress on, 2014, pp. 341-346.
[29] N. Chen, X. Wang, X. Men, X. Han, J. Sun, and C. Guo, "Hybrid BCI based control strategy of the intelligent wheelchair manipulator system," in 2018 13th IEEE Conference on Industrial Electronics and Applications (ICIEA), 2018, pp. 824-828.

[30] M. Samadi and M. F. Othman, "Global Path Planning for Autonomous Mobile Robot Using Genetic Algorithm,” in 2013 International Conference on Signal-Image Technology Internet-Based Systems, 2013, pp. 726-730.

[31] Al-Jadid M. Disability in Saudi Arabia. Saudi Med J. 2013;34(5):453-60.

[32] B. R. K. Mantha, C. C. Menassa, V. R. Kamat, and C. R. D’Souza, "Evaluation of Preference- and Constraint-Sensitive Path Planning for Assisted Navigation in Indoor Building Environments,” J. Comput. Civ. Eng., vol. 34, no. 1, p. 04019050, Jan. 2020, doi: 10.1061/(ASCE)CP.1943-5487.0000865.

[33] "EMOTIV Insight Brainwear ${ }^{\circledR} 5$ Channel Wireless EEG Headset." [Online]. Available: https://www.emotiv.com/insight/. [Accessed: 07April-2021].

[34] S. G. Hart and L. E. Staveland, "Development of NASA-TLX (Task Load Index): Results of empirical and theoretical research," in Advances in psychology, vol. 52, Elsevier, 1988, pp. 139-183.

[35] L. Bi, F. xin'an, and Y. Liu, "EEG-Based Brain-Controlled Mobile Robots: A Survey," Human-Machine Systems, IEEE Transactions on, vol. 43, pp. 161-176, Mar. 2013, doi: 10.1109/TSMCC.2012.2219046.

[36] S. Desai, S. Mantha, and V. Phalle, Advances in smart wheelchair technology. 2017, p. 7. 